\title{
Instability of the Chaotic ENSO: The Growth-Phase Predictability Barrier
}

\author{
ROGER M. SAMELSON \\ College of Oceanic and Atmospheric Sciences, Oregon State University, Corvallis, Oregon \\ ELI TZIPERMAN \\ Weizmann Institute of Science, Rehovot, Israel
}

(Manuscript received 5 July 2000, in final form 21 May 2001)

\begin{abstract}
The local predictability of the El Niño-Southern Oscillation (ENSO) is examined by the analysis of the evolution of small disturbances to an unstable 4.3-yr ENSO cycle in the Cane-Zebiak model forced by perpetual July conditions. The 4.3-yr cycle represents the dominant near-recurrent behavior in this weakly chaotic regime, so analysis of this single cycle gives useful insights into the dynamics of the irregular oscillation. Growing and neutral time-dependent eigenmodes of the unstable cycle are computed. Disturbance growth analyses based on these eigenmodes, and on singular vectors computed in the unstable-neutral subspace, suggest that there is a predictability barrier associated with the growth phase of El Niño conditions. This barrier arises because the growth mechanism for disturbances to the cycle is nearly the same as the growth mechanism for the El Niño conditions themselves. The local amplification of disturbances during the growth phase is several times greater than the eigenmode amplification associated with time-dependent (Floquet) normal-mode instability of the cycle. It is suggested that the existence of an ENSO predictability barrier tied to the growth phase of El Niño conditions is likely a robust result, independent of the particular model.
\end{abstract}

\section{Introduction}

Recent studies of El Niño-Southern Oscillation's (ENSO's) irregularity and hence limited predictability have focused on two possible mechanisms: self-sustained variability made irregular by low-order chaos driven by the seasonal cycle (Tziperman et al. 1994, 1995; Jin et al. 1994; Chang et al. 1994), or damped oscillatory behavior randomized by the amplification of atmospheric noise through the nonnormal dynamics of the equatorial Pacific (Farrell and Ioannou 1996; Penland and Sardeshmukh 1995; Moore and Kleeman 1996, 1997). The limited length of time series from presently available data is not sufficient to distinguish between the two scenarios. It has been proposed that ENSO may have been self-sustained (possibly chaotic) during some decades and damped during other decades. It is important, therefore, to examine the detailed predictability implications of each of the two alternatives for ENSO's irregularity, in the hope that differences between the two scenarios will eventually arise that will be possible to verify by analyzing the available data.

Corresponding author address: Dr. Roger M. Samelson, College of Oceanic and Atmospheric Sciences, Oregon State University, 104 Ocean Administration Building, Corvallis, OR 97331-5503.

E-mail: rsamelson@oce.orst.edu
A number of previous studies have addressed the seasonal variability in ENSO's predictability, also known as the "spring barrier," for model regimes in which ENSO is damped, self-sustained, and chaotic (Webster and Yang 1992; Torrence and Webster 1998; Weiss and Weiss 1999; Xue et al. 1994; Chen et al. 1995; Moore and Kleeman 1996; Chen et al. 1997). However, there has been much less effort to study the variability of the inherent ENSO predictability as function of the phase within the ENSO cycle. That is, is ENSO more predictable during the growth phase of El Niño warm events, or during La Niña events, etc.? By "inherent predictability" we mean the limited predictability due to the ENSO dynamics, rather than due to the limits on the available data for initializing ENSO predictions. Moore and Kleeman (1996) and Chen et al. (1997) have examined this issue in a stable oscillatory regime by calculating optimal amplification in two different intermediate coupled ocean-atmosphere ENSO models. Their results have been quite different, with Moore and Kleeman (1996) finding that ENSO is least predictable during its growth phase, and Chen et al. (1997) that the maximum growth rate occurs at the transition from a cold event to a warm event. The spatial structures found in these two studies for the optimal perturbation patterns are also quite dramatically different.

Here, we investigate the variability of ENSO's in- 
herent predictability along the ENSO cycle, for the first time under the scenario (Vallis 1986; Tziperman et al. 1994; Jin et al. 1994; Chang et al. 1994) in which ENSO's predictability is limited by low-order chaos. We do this by analyzing results from the Cane-Zebiak model (hereafter CZ model; Zebiak and Cane 1987) whose irregular ENSO events have been shown to be due to low-order chaos driven by the seasonal cycle (Tziperman et al. 1994, 1995). The CZ model was also shown to be dominated by low-order chaos even in the absence of seasonal forcing (Tziperman et al. 1997), which is the case analyzed in this work. This predictability analysis in a chaotic regime poses a special challenge as compared to the nonchaotic case as the model does not display a regular ENSO cycle which may be easily analyzed as function of the ENSO phase. Instead, the model solution appears to jump between various unstable periodic solutions (orbits). Our analysis method here is based on extracting a single dominant unstable periodic orbit from the chaotic ENSO attractor (Tziperman et al. 1997) and analyzing the predictability along this unstable orbit. A similar approach was taken by Samelson (2001) who analyzed the growth of disturbances to stable and unstable, nonlinear periodic Rossby wave solutions in an idealized model of baroclinic instability.

The main result of this paper is that ENSO is least predictable during the growth phase of warm El Niño events, even in the absence of seasonality in the model forcing or specified background fields. Similar results were found in a nonchaotic regime by Moore and Kleeman (1996). We refer to this phenomenon as the "growth-phase predictability barrier." We see this growth phase barrier as complementing the "spring predictability barrier" previously found in both chaotic and nonchaotic regimes. The spring predictability barrier is believed to be a result of the seasonality in the strength of the coupled ocean-atmosphere coupling and thus strength of the coupled instability leading to the development of ENSO events. We analyze here the physical mechanism of the growth phase predictability barrier (with all seasonal information removed from the model dynamics) and show that the predictability is limited by essentially the same dynamics that are responsible for ENSO's growth, irrespective of the seasonal cycle. Given that the growth phase of ENSO is locked to the spring and early summer (Tziperman et al. 1998; Galanti and Tziperman 2000), we speculate that the spring predictability barrier found in previous studies may be, at least partially, a manifestation of the growth-phase barrier proposed here, rather than a purely seasonal-related signal as previously suggested.

In the following, we present an eigenmode analysis of the instability of an ENSO cycle (section 2), proceed to a study of the predictability along the cycle, which includes consideration of the nonnormal nature of the dynamics (section 3), and conclude in section 4 .

\section{Eigenmode analysis of ENSO-cycle instability}

a. A 4.3-yr ENSO cycle

While the irregularity of the standard CZ model, using a seasonally varying specified background state, has been shown to be driven by the seasonal cycle according to the quasi-periodicity route to chaos (Tziperman et al. 1994, 1995), the model is also weakly chaotic with a perpetual-July background state. Previous work has begun to analyze these irregular oscillations in terms of unstable periodic orbits (UPOs) associated with the chaotic attractor (Tziperman et al. 1997). These orbits are exactly periodic solutions of the nonlinear model equations. When the model state approaches one of them, the model oscillates in an approximately periodic fashion for some time. Because the UPOs are also unstable, the model state eventually departs from any such cycle, and the model oscillations take on a significantly different character, until the nonlinear evolution eventually brings the model solution near the given UPO once again.

The UPOs are interesting and relevant physical objects whose dynamics may be directly analyzed. They may closely approximate the evolution of the model state for substantial intervals of time (whose extent depends on the strength of the instability of the UPOs), and present a convenient starting point for the study of the irregular oscillations. The perpetual-July CZ model is especially appropriate for the purposes of the present study for two reasons. First, the lack of seasonality allows us to differentiate predictability variations along the ENSO cycle from seasonal predictability effects studied previously. Second, the weakly chaotic nature induced by this particular model background allows us to extract and analyze the model UPOs more easily.

The CZ model with perpetual-July conditions has a dominant near-recurrent behavior with a period of 4.3 yr. This behavior is associated with the existence of a UPO with this same period. It has recently been shown (Tziperman et al. 1997) that the departure of the model state from this UPO can be inhibited by applying carefully chosen perturbations to a single model variable (oceanic Kelvin wave amplitude) at a single point in longitude (the western boundary of the Pacific Ocean) that effectively stabilize the UPO, resulting in an essentially periodic model oscillation that closely follows the UPO of the original chaotic model (this procedure amounts to a "control of chaos" in the CZ model).

Here, we analyze the dynamics and predictability of this unstable 4.3-yr cycle. We use a long (100 000 yr) time series of the amplitudes of the first six multivariate empirical orthogonal functions (EOFs) of the CZ model SST, thermocline depth, and zonal wind velocity fields. The time series increment $\delta t$ is $1 / 3$ of a month, equal to the integration time-step of the $\mathrm{CZ}$ model. The multivariate EOFs are calculated from model covariances during a 500-yr run, and model variables from a long model run are then projected onto these multivariate 
EOFs to generate the long EOF amplitude time series. This (standard) procedure avoids the unmanageably large storage requirements that would be required to compute EOFs from the long series directly. Before the calculation of the EOFs, the SST, zonal wind and thermocline depth fields were normalized to have the same root mean squared variability, so that they would all contribute equally to the multivariate EOFs. The EOFs of the $\mathrm{CZ}$ model have been published elsewhere so we do not show them here, but we do note that the structure of the first EOF is basically that of a mature El Niño warming event, as this will feature in the following.

The UPO and its stability are investigated in the sixdimensional phase space of the six EOF amplitudes. Only six EOFs are necessary to describe the dominant variability in this weakly chaotic, perpetual-July case; more EOFs would be required for the case with seasonal forcing (e.g., Xue et al. 1994). Not only do we need to represent a simpler system (perpetual July) than the full CZ model, but note also that we only need a representation of this simpler system around a single UPO, which further reduces the required degrees of freedom. Furthermore, the previous successful application of chaos control methods to the same perpetual-July model using a three-dimensional, delay-coordinate phase space (Tziperman et al. 1997), also indicates that only a few degrees of freedom are needed to represent the model dynamics in this parameter regime. Certainly using six EOFs, which is an optimally efficient representation of the dynamics, should suffice if three suboptimal delay coordinates were sufficient in the above mentioned chaos-control study. Overall, it seems that the properties of the UPO identified in the present six-EOF-reduced representation correspond to those of a true UPO of the CZ model itself, so that no additional EOFs are needed for our purposes.

To identify the UPO, we search for near recurrences in the long time series. Let $\mathbf{X}(t)$ be the vector of the six EOF amplitudes $X_{j}, j=1, \ldots, 6$ at time $t$ :

$$
\mathbf{X}(t)=\left[X_{1}(t), X_{2}(t), \ldots, X_{6}(t)\right] .
$$

Then $\mathbf{X}(t)$ may be regarded as a point in the six-dimensional phase space of EOF amplitudes. Given a pe$\operatorname{riod} p$, we search for phase space points $\mathbf{X}(t)$ that return to the same neighborhood after a period $p$, so that

$$
\begin{aligned}
& \|\mathbf{X}(t+p)-\mathbf{X}(t)\| \\
& \quad=\left\{\sum_{i=1}^{6}\left[X_{i}(t+p)-X_{i}(t)\right]^{2}\right\}^{1 / 2}<\varepsilon
\end{aligned}
$$

for some small $\varepsilon$. When the number of such "near returns" is plotted as a function of $p$, the dominant UPOs show up as peaks. There are two such dominant UPOs in the perpetual July CZ model, at $p=4.3$ and $p=7.8$ yr (Tziperman et al. 1997). The 4.3-yr UPO is approximated in the present analysis by the single nearest return occurring near this period in the long time series.

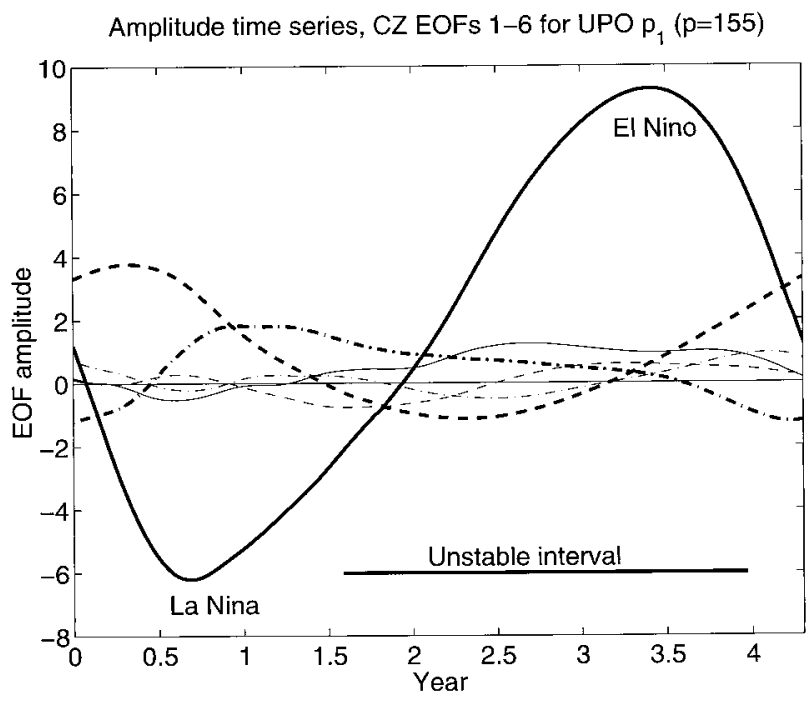

FIG. 1. The 4.3-yr unstable cycle (UPO). Time series of EOF components along the UPO: EOF 1 (thick solid line), EOF 2 (thick dashed), EOF 3 (thick dash-dot), EOF 4 (thin solid), EOF 5 (thin dashed), EOF 6 (thin dash-dot). The period of disturbance growth identified in this study is indicated by the horizontal line labeled "Unstable interval." The approximate times of the El Niño and La Niña maxima on the 4.3-yr cycle are labeled.

That is, the UPO is represented by the time series segment from $\mathbf{X}(t)$ to $\mathbf{X}(t+p)$, where $\|\mathbf{X}(t+p)-\mathbf{X}(t)\|$ is the smallest occurrence in the entire time series. This nearest return UPO has a period of $n_{p}=155$ time-series points or $p=n_{p} \delta t \approx 4.3 \mathrm{yr}$, in agreement with the result obtained by searching for the period with the maximum number of near returns. An alternate approximation to the UPO based on averaging over the location of many near returns is discussed below. The high dimension (order 30000 variables) of the $\mathrm{CZ}$ model prevents a straightforward application of direct solution methods.

The nearest-return approximation to the 4.3-yr UPO is shown in Fig. 1. It is dominated by an oscillation in the first EOF amplitude, corresponding to a full ENSO cycle. This is a somewhat weak event, and it is different from the observed ones in that the El Niño and La Niña have roughly equal amplitudes, but otherwise this ENSO cycle is reasonably representative of events in the CZ model as well as in reality (Neelin et al. 1998). Maximum El Niño conditions occur near 3.4 yr, and maximum La Niña conditions near 0.7 yr. The transition from El Niño to La Niña is much more rapid than the reverse. Zero crossings of the first EOF occur near 0.1 yr and 2 yr. The growth phase of El Niño, which will feature prominently in the following analysis, is roughly the period from 2 to $3.4 \mathrm{yr}$. The $p=7.8$-yr UPO corresponds to a less realistic ENSO cycle, which involves a small-amplitude event followed by a large-amplitude event, forming together a cycle that is roughly twice the period of observed ENSO events. Since this cycle is less realistic, we do not analyze it in detail, and expect 
that the lessons to be learned from the 4.3-yr cycle would be more relevant to the observed events.

In the following, we focus on the analysis of this single UPO. A qualitative examination of the original time series indicates that the system spends at least $25 \%$ of the time in relatively small amplitude, nearly symmetric oscillations that resemble the 4.3-yr cycle (Tziperman et al. 1997). It often undergoes a few such cycles before leaving this UPO and oscillating in a manner that is characteristic of the second dominant UPO. The dominance of the 4.3-yr cycle, indicates that its consideration may be sufficient to provide substantial insight into the $\mathrm{CZ}$ chaotic dynamics. This is consistent with previous experience for weakly chaotic systems, for which much of the basic structure of the attractor can often be obtained from the lowest-order cycle (e.g., Samelson 2001). A more complete description would include analysis of the higher-order unstable cycles as well, following the periodic orbit expansion theories that have been developed for low-order systems and have begun to be extended to more complex systems (Artuso et al. 1990a,b; Christiansen et al. 1997; Cvitanović et al. 2000; Zoldi and Greenside 1998). However, the high dimension of the $\mathrm{CZ}$ model makes the search for higherorder cycles challenging.

\section{b. Stability analysis-Method}

UPOs such as the 4.3-yr cycle shown above are perfectly periodic and hence (in principle) perfectly predictable solutions of the nonlinear model equations, corresponding to a full ENSO cycle. However, because the UPOs are unstable, nearby model solutions do not remain near them for long periods of time, but instead eventually depart to other regions of phase space. This instability limits the predictability of the system.

The rate of departure from the UPO need not be, and often is not, uniform along the UPO. In this and the next section, we calculate the amplification rate of deviations of the model state from the UPO, as a function of location along the UPO. To do this, we fit linear maps to the local model dynamics along the UPO, and study how small deviations from the UPO evolve under the action of these local maps. We then use these results to develop an estimate of predictability along the UPO. Our analysis is essentially a simplified approach to the standard problem of Floquet theory for analyzing the stability of periodic solutions (Coddington and Levinson 1955); for geophysical applications, see, for example, Jin et al. (1996), Strong et al. (1995), and Samelson (2001).

Denote the nearest-return estimate of the 4.3-yr UPO, described in the previous subsection, by $\left\{\mathbf{Y}\left(t_{k}\right), k=1\right.$, $\left.\ldots, n_{p}\right\}$. For each point $\mathbf{Y}\left(t_{k}\right)$ on the UPO, we identify the subset of data points $\left\{\mathbf{X}\left(t_{j}\right), j=1, \ldots, J\right\}$ in the long EOF amplitude time series $\mathbf{X}(t)$, for which the trajectory begins within $\varepsilon$ of $\mathbf{Y}\left(t_{k}\right)$ for some $t_{k}$, and returns to within $\varepsilon$ of the same point $\mathbf{Y}\left(t_{k}\right)$ after a time interval equal to the UPO period $p$. That is, we identify those points for which

$$
\begin{aligned}
\left\|\mathbf{X}\left(t_{j}\right)-\mathbf{Y}\left(t_{k}\right)\right\| & <\varepsilon, \quad \text { and } \\
\left\|\mathbf{X}\left(t_{j}+p\right)-\mathbf{Y}\left(t_{k}\right)\right\| & <\varepsilon .
\end{aligned}
$$

Using this set of $J$ near-return pairs $\left[\mathbf{X}\left(t_{j}\right), X\left(t_{j}+p\right)\right]$, we fit a linear map $\mathbf{M}\left(t_{k}\right)$ that maps the deviation of a nearby state from $\mathbf{Y}\left(t_{k}\right)$ into a deviation from $\mathbf{Y}\left(t_{k}\right)$ after a time interval equal to one period of the UPO. For each pair, we want

$$
X_{i}\left(t_{j}+p\right)-Y_{i}\left(t_{k}\right)=\sum_{l=1}^{6} M_{i l}\left(t_{k}\right)\left[X_{l}\left(t_{j}\right)-Y_{l}\left(t_{k}\right)\right] .
$$

Defining $a^{j}{ }_{i}=X_{i}\left(t_{j}+p\right)-Y_{i}\left(t_{k}\right)$ and $b^{j}{ }_{l}=X_{l}\left(t_{j}\right)-$ $Y_{l}\left(t_{k}\right)$ to be the $j$ th deviation vectors, (3) may be written as $a^{j}{ }_{i}=\sum^{6}{ }_{l=1} M_{i l} b^{j}{ }$. Since (3) is to hold for all $J$ nearreturn pairs (typically $J \approx 2000$ here), the elements of $M$ are over-determined, and (3) cannot be satisfied exactly for all $j$. We compute a least-square fit by minimizing $\mathcal{J}=\Sigma_{i, j}\left(a^{j}{ }_{i}-\Sigma_{l} M_{i l} b^{j}{ }_{l}\right)^{2}$ with respect to the coefficients $M_{i l}$. At the minimum of $\mathcal{I}$, we have $\partial \mathscr{I} / \partial M_{i l}$ $=0$, which results in the equation,

$$
\sum_{j=1}^{J} a_{i}^{j} b_{l}^{j}=\sum_{n=1}^{6} M_{i n}\left[\sum_{j=1}^{J} b_{n}^{j} b_{l}^{j}\right] .
$$

Defining

$$
A_{i l} \equiv \sum_{j} a_{i}^{j} b_{l}^{j} ; \quad B_{n l} \equiv \sum_{j} b_{n}^{j} b_{l}^{j},
$$

we get the equation for the linear map in the matrix form $\mathbf{A}=\mathbf{M}\left(t_{k}\right) \mathbf{B}$, whose solution for $\mathbf{M}\left(t_{k}\right)$ (accomplished using singular value decomposition) gives the best-fit linear map. In order to improve numerical conditioning, we carry out this procedure using EOF time series $X_{i}(t)$ that have been normalized by the difference of their maximum and minimum values.

At each point $t_{k}$ on the UPO, we then solve the eigenvalue problem

$$
\mathbf{M}\left(t_{k}\right) \mathbf{u}\left(t_{k}\right)=\Lambda \mathbf{u}\left(t_{k}\right)
$$

for the eigenvalues $\Lambda_{j}$ and eigenvectors $\mathbf{u}_{j}$. The $\Lambda_{j}$ are the characteristic Floquet multipliers and should in principle be independent of $t_{k}$. We normalize the $\mathbf{u}_{j}$ so that $\left\|\mathbf{u}_{j}\right\|=1$.

A second numerical estimate of the $\Lambda_{j}$ and $\mathbf{u}_{j}$ can be obtained from "partial-period" $\operatorname{maps} \mathbf{M}^{p}\left(t_{k} ; t_{k}+m\right)$, which describe the evolution of linear disturbances from point $t_{k}$ on the UPO (1) to point $t_{k}+m$ on the same UPO. We fit these partial-period maps $\mathbf{M}^{p}$ using essentially the same method as above. In this case, the initial and final (base and image) points for the map are two different points along the cycle, separated by an interval of $n_{m}$ points (time interval $m=n_{m} \delta t<p$ ). Thus, for each pair with 


$$
\begin{aligned}
\left\|\mathbf{X}\left(t_{j}\right)-\mathbf{Y}\left(t_{k}\right)\right\| & <\varepsilon, \quad \text { and } \\
\left\|\mathbf{X}\left(t_{j}+m\right)-\mathbf{Y}\left(t_{k}+m\right)\right\| & <\varepsilon,
\end{aligned}
$$

we want

$$
\begin{aligned}
& X_{i}\left(t_{j}+m\right)-Y_{i}\left(t_{k}+m\right) \\
& \quad=\sum_{l=1}^{6} M_{i l}^{p}\left(t_{k} ; t_{k}+m\right)\left[X_{l}\left(t_{j}\right)-Y_{l}\left(t_{k}\right)\right],
\end{aligned}
$$

in place of (3). Now the elements of $\mathbf{M}^{p}$ are over determined, and again we obtain the map by a least squares fit. For a sequence of $S$ points along the cycle, $t_{1}, \ldots$, $t_{s}$, we fit a sequence of $S$ linear maps that describe the evolution of linear disturbances from point to point in this sequence along the cycle. A composite full-period return map $\mathbf{M}^{c}\left(t_{1}\right)$ may be computed by composing all the maps in the sequence,

$$
\begin{aligned}
\mathbf{M}^{c}= & \mathbf{M}^{p}\left(t_{S} ; t_{1}\right) \times \mathbf{M}^{p}\left(t_{S-1} ; t_{S}\right) \times \cdots \times \mathbf{M}^{p}\left(t_{2} ; t_{3}\right) \\
& \times \mathbf{M}^{p}\left(t_{1} ; t_{2}\right) .
\end{aligned}
$$

We then can solve the equivalent of the eigenvalue problem (4) for $\mathbf{M}^{c}$. This gives a second estimate of the $\Lambda_{j}$ and $\mathbf{u}_{j}$.

The eigenvalues and eigenvectors of $\mathbf{M}$ and the composite return map $\mathbf{M}^{c}$ should in principle be equal, but this is never exactly the case for these fitted maps. We find the composite return map fits generally to be more stable than the full-period return map fits, and primarily use these in the following. In the following we take $n_{m}$ $=9$, corresponding to a partial-period map interval of $m=n_{m} \delta t=3$ months. In that case, the sequence $t_{1}$, $\ldots, t_{s}$ consists of 17 points $(S=17)$; the last interval $\left(t_{s}, t_{1}\right)$ has map interval $n_{m}=11$ instead of 9 , to close the $n_{p}=155$ cycle. We have computed fits for many different values of $n_{m}$, and found $n_{m}=9$ to provide an optimal combination of fit stability and temporal resolution.

Finally, note that the unstable and stable eigenvectors of the full period return map define local approximations to the unstable and stable manifolds of the UPO. That is, a perturbation to the trajectory along the direction in phase space of the unstable eigenvector leads to an exponentially growing departure of the disturbance from the UPO. A perturbation along the stable manifold, on the other hand, results in an exponential decay of the disturbance. Each UPO must have at least one unstable $\left(\left|\Lambda_{j}\right|>1\right)$ eigenvector, one neutral $\left(\Lambda_{j}=1\right)$ eigenvector that is everywhere tangent to the UPO itself (since two solutions that differ only in their initial phase along the UPO will have exactly the same phase difference after one oscillation period), and (since the $\mathrm{CZ}$ model is a dissipative system) at least one stable $\left(\left|\Lambda_{j}\right|<1\right)$ eigenvector.

\section{c. Stability analysis-results}

The composite return maps $\mathbf{M}^{c}\left(t_{k}\right)$ consistently show a single unstable eigenvalue $\Lambda_{1} \approx-1.8$ (with corre- sponding eigenvector $\mathbf{u}_{1}$ ), a "neutral" eigenvalue $\Lambda_{2}$ that is close to its expected value of one (eigenvector $\mathbf{u}_{2}$ ), and four smaller eigenvalues (Fig. 2a). The neutral eigenvector $\mathbf{u}_{2}$ is nearly tangent to the UPO, as it must be. The unstable eigenvector $\mathbf{u}_{1}$ is roughly tangent to the large-scale transverse structure of the attractor (Fig. 3 ), as expected for relatively simple attractor geometries; physically this means that if the model oceanatmosphere state is changed by adding a small disturbance with the physical structure of the unstable eigenvector, the result is a perturbed state that would eventually arise naturally as the system evolved in time, or at least is very close to such a state. That is, the disturbance does not push the model ocean and atmosphere into an inaccessible or "artificial" state that would not normally occur except during transient adjustment from specific initial conditions. Along with the unstable and neutral eigenvectors, the algorithm produces four stable $\left(\Lambda_{j}<1\right)$ eigenvectors. In contrast to the unstable and neutral vectors, these estimates of stable vectors are not reliable, for reasons discussed below. Consequently, we do not consider the stable vectors further.

The components of the unstable and neutral eigenvectors correspond, by (1), to amplitudes of the first six EOFs of the CZ model. These components vary smoothly as a function of location along the cycle (Fig. 4). The first EOF component dominates both the unstable and the neutral eigenvectors over most of the cycle. The second EOF component of the unstable eigenvector is large during the La Niña maximum $(t \approx 0.5 \mathrm{yr})$, and the second and third EOF components of the neutral eigenvector are large during both the La Niña and El Niño $(t \approx 3.5 \mathrm{yr})$ maxima. Since $\Lambda_{1}<0$, the unstable eigenvector components change sign after one cycle, as indicated in Fig. 4a by the opposing signs of the components at $t=0$ and $t=155 \delta t$. The unstable and neutral eigenvectors are nearly parallel where both eigenvectors are dominated by the first EOF component, and nearly orthogonal near the La Niña and El Niño maxima (Fig. $4 c$ ). The similarity of the unstable and neutral eigenvectors during most of the El Niño growth phase (1.5$3.5 \mathrm{yr}$ ) suggests that the mechanism for unstable disturbance growth is essentially the same as the mechanism for growth of El Niño conditions in the basic oscillation. The dynamics and physical structure of these eigenvectors are discussed further below (section 2d).

The map-fitting procedure produces reliable estimates of the unstable and neutral eigenvectors and eigenvalues. We have tested the reliability and convergence of these estimates, and their dependence on the number of EOFs amplitudes used in the fit and on other fitting parameters, by comparing the eigenvalues and eigenvectors of the composite return map $\mathbf{M}^{c}$ along the cycle for many different fits (e.g., Fig. 2), and by comparing the eigenvectors at points along the cycle to the distribution of nearby phase-space points on the attractor from the EOF time series $\mathbf{X}(t)$ (e.g., Fig. 3). As mentioned above, the eigenvalues $\Lambda_{j}$ should be independent 
(a)

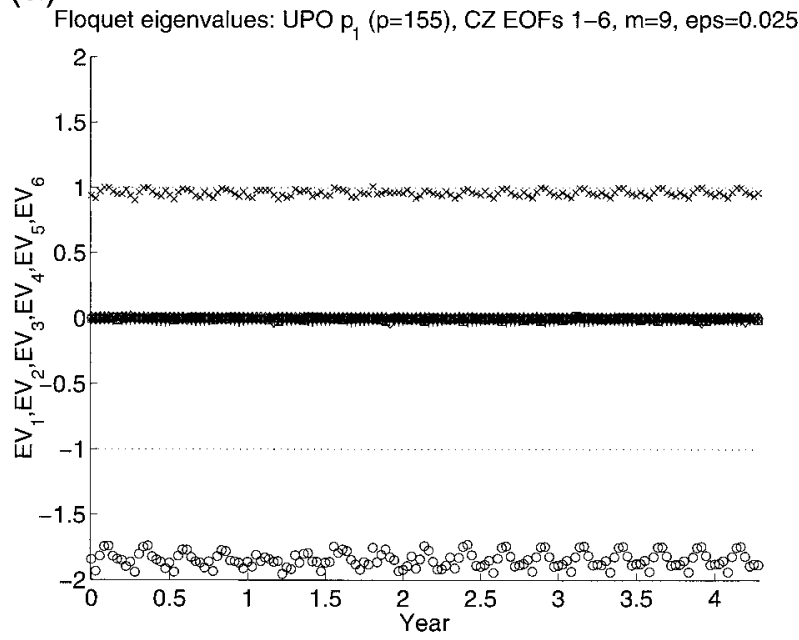

(b)

Floquet eigenvalues: UPO $p_{1}(p=155)$, CZ EOFs $1-3, m=155$, eps $=0.075$

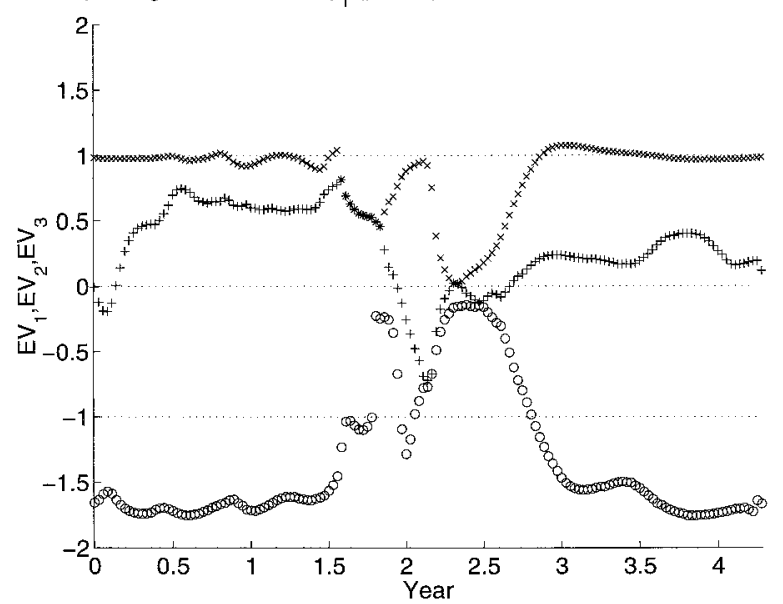

(c)

Floquet eigenvalues: UPO $p_{1}(p=155), C Z$ EOFs $1-6, m=155$, eps $=0.075$

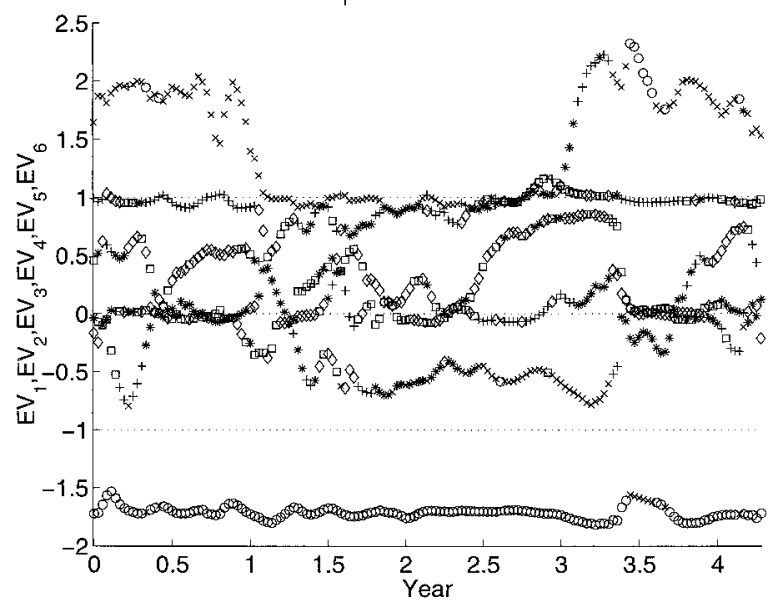

FIG. 2. Floquet multipliers $\Lambda_{j}$ vs time along the UPO for three different sets of map fitting parameters. The results in (a) are for the composite full-return maps constructed from the corresponding partial-period maps, which provide the best estimates of the unstable
Poincare section: UPO $p_{1}(p=155)$, CZ EOFs $1-6, m=9, j=100$

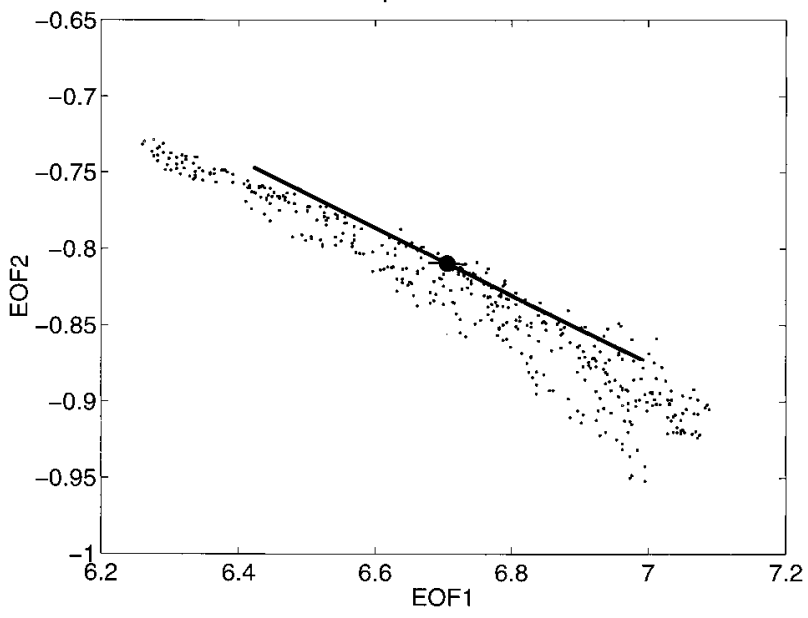

FIG. 3. Projection in the EOF 1-EOF 2 plane of Poincare section of attractor normal to the UPO in Fig. 1 at time $t_{k}=99 \delta t=2.75$ yr. The UPO intersects the plane at the solid circle. The projection of the unstable Floquet eigenvector $\mathbf{v}_{1}$ is indicated by the thick solid line. The projection of the neutral eigenvector $\mathbf{v}_{2}$ with equal amplitude, which would vanish if $\mathbf{v}_{2}$ were exactly equal to the true neutral eigenvector, is indicated by the thin solid line.

of $t_{k}$. Their dependence on $t_{k}$ arises from the map-fitting procedure and thus serves as a qualitative measure of the reliability of the fits.

For directly fitted full-period return maps $M\left(t_{k}\right)$, we find that this dependence is strong along certain segments of the cycle when only three or four EOF time series are used in the fitting procedure (Fig. 2b). A stable estimate of the leading eigenvalue $\Lambda_{1}$ can be obtained from a full-period map with five or six EOFs, but additional, spurious eigenvalues then arise (Fig. 2c). The main difficulty in using the full-period maps is that the cycle is sufficiently unstable that initially nearby points often have moved far from the cycle after one period, and so must be discarded during the fitting procedure. This reduces the number of points available for the fit, and forces the fit to depend more on very nearby points that move very slowly, increasing its susceptibility to contamination from noise from a number of sources, including our imperfect estimate of the cycle itself. To obtain a stable estimate of the second eigenvalue $\Lambda_{2}$, it is necessary to use the composite partial-period maps $M^{c}\left(t_{k}\right)$ and five or six EOFs (Fig. 2a). The stability of these eigenvalue estimates along the cycle (Fig. 2a), and for moderate changes in fitting parameters, and the ap-

$\leftarrow$

and neutral eigenvectors and eigenvalues. The results in (b) and (c), for directly fitted full-return maps, are shown for comparison, to illustrate the variations along the cycle that can arise in the computed eigenvalues, indicating noise in the fitting procedure. In (a), $m=9$ (partial-period maps with 3-month mapping interval), 6 EOFs; in (b), $m=155$ (full-period maps), 3 EOFs; and in (c), $m=155$ (fullperiod maps), 6 EOFs. 
(a)

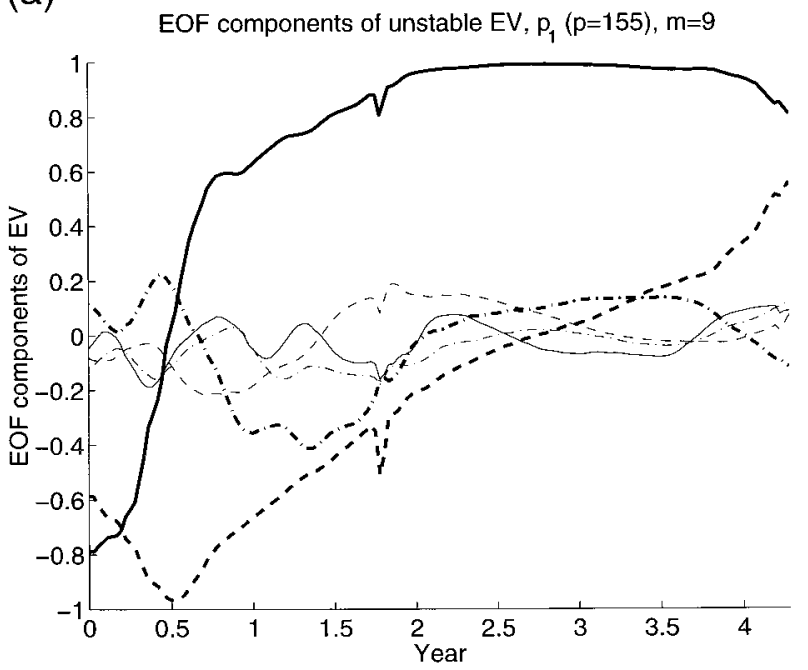

(b)

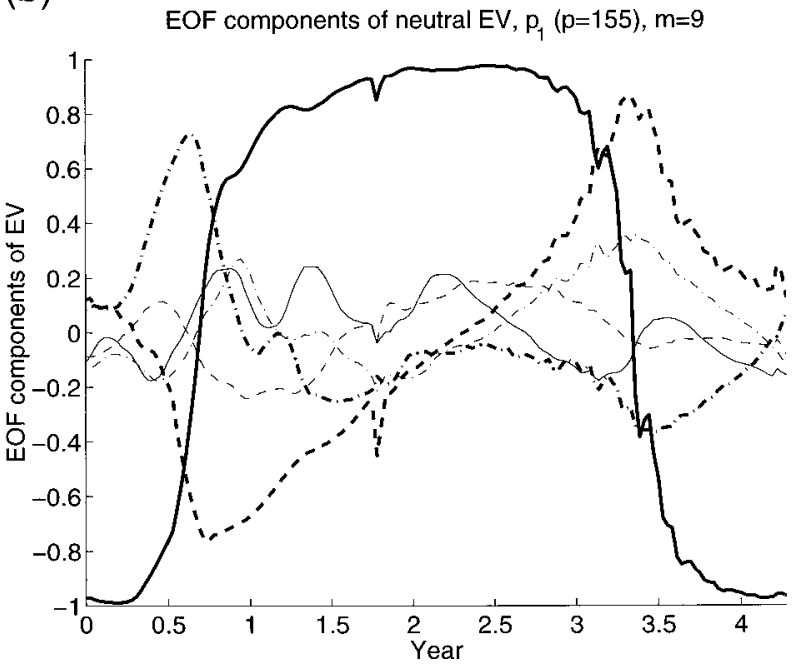

(c)

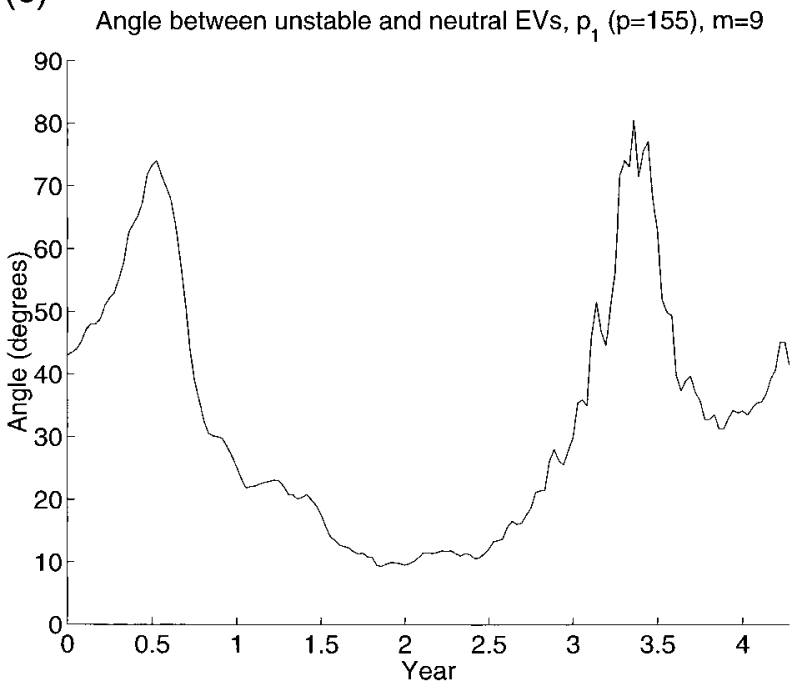

parent absence of noise in the eigenvector component time series (Fig. 4) suggests that these fits are reliable, and that a five- or six-dimensional phase space is sufficient to represent the perpetual July CZ model dynamics around the UPO.

The difficulty in estimating the stable eigenvectors arises entirely from the distribution of phase-space points $\mathbf{X}(t)$ near the attractor (Fig. 3), which is in turn a result of the model dynamics. Because the attractor is compressed along the stable eigenvectors, the distance along the stable eigenvector of the points $\mathbf{X}(t)$ from the attractor is always essentially zero, that is, small enough that it is dominated by noise. Thus, these points do not show a numerically significant contraction along the stable eigenvectors over the mapping intervals. Consequently, empirical map-fitting procedures of the present type cannot be used to estimate reliably the corresponding contraction rates and directions, and thus the stable eigenvalues and eigenvectors.

\section{Local predictability \\ a. Eigenvector amplification}

In order to study local predictability along the cycle, we use the partial-period maps, $\mathbf{M}^{p}$, to compute the local amplification of linear disturbances over different segments of the cycle. We compute local amplifications in two ways. First, in this section, we compute the local amplification of disturbances along either the unstable or the neutral eigenvectors of the full-return composite maps, which are the directions of the unstable and neutral manifolds of the UPO. Second, in the next section, we compute singular vectors (optimal disturbances; e.g., Farrell and Ioannou 1996) for disturbances in the subspace spanned by the unstable and neutral vectors. We have excluded the stable vectors from these analyses because of the unreliability of the stable-vector fits (section $2 \mathrm{c}$ ), and because the relatively narrow distribution of points along the stable vector on the attractor indicates that these directions are not sampled by the natural evolution of the model and do not represent accessible phase space directions in which prediction errors may develop. Clearly an addition of noise to the system may change this by sampling also these stable directions, but we restrict the analysis in this paper to the purely deterministic dynamics of the CZ model, and focus on the case in which the only error relevant to the prediction problem is the error in the specification of the initial position of the state on the attractor.

FIG. 4. EOF components of eigenvector vs point along cycle for $m=9$ for (a), the unit unstable eigenvector $\mathbf{u}_{1}$; and (b), the unit neutral eigenvector $\mathbf{u}_{2}$. The components are EOF 1 (thick solid line), EOF 2 (thick dashed), EOF 3 (thick dash-dot), EOF 4 (thin solid), EOF 5 (thin dashed), EOF 6 (thin dash-dot). (c) The angle across $\left(\mathbf{u}_{1} \cdot \mathbf{u}_{2}\right)$, in degrees. 
We compute the local amplification of the unstable eigenvector over 3-month $\left(n_{m}=9, m=9 \delta t\right)$ intervals along the UPO as follows. At each point $t_{k}$ along the UPO, we compute the image $\mathbf{u}^{*}\left(t_{k}+m\right)=\mathbf{M}^{p} \mathbf{u}_{1}\left(t_{k}\right)$ of the eigenvector $\mathbf{u}_{1}$. Then, since $\left\|\mathbf{u}_{1}\right\|=1$, the norm of the image $\mathbf{u}^{*}$ gives the 3-month amplification factor, $\left\|\mathbf{u}^{*}\right\| /\left\|\mathbf{u}_{1}\left(t_{k}\right)\right\|=\left\|\mathbf{u}^{*}\right\|$ as a function of $t_{k}$. In general, the results of the local predictability analysis can be expected to depend on which norm is chosen. We use primarily the standard Euclidean norm $\|\cdot\|$ in the EOF amplitude phase space, as in (2). The standard norm is based directly on the EOFs, and so provides a natural quantification of significant physical variability. Other choices are also possible, and could lead to different results, as discussed below. We have examined the sensitivity of our results to the choice of norm and found that results with other physically motivated norms are roughly consistent with those obtained using the standard Euclidean norm.

The results for the amplification factors $\left\|\mathbf{u}^{*}\right\|$ show that disturbances along the unstable eigenvector are amplified over 3-month intervals primarily during the period 1.5-3.5 yr (Fig. 5a). For the UPO itself, this period corresponds closely to the growth phase of El Niño conditions (Fig. 1). There is also a brief period of amplification of the unstable vector during the La Niña maximum, near 0.7 yr (Figs. 5a,1), and a brief period of very weak amplification near 1.4 yr. Disturbances along the neutral eigenvector grow rapidly, over 3month intervals, during the period 3.2-4 yr, the maximum and early decay phase of El Niño conditions, and less rapidly during 1.7-2.2 yr the early El Niño onset phase. There are also two short intervals of weak neutral-disturbance growth following the La Niña maximum.

The cumulative linear growth of unit-norm disturbances along the unstable or neutral eigenvectors during the course of a complete cycle of the UPO may be estimated by computing products of these 3-month amplification factors from succeeding maps along the sequences $t_{1}, \ldots, t_{S}$ of points defined above (Figs. 5b,c). Disturbances along the unstable eigenvector that are initially of unit amplitude during the first two years of the cycle all amplify by a factor of $3-5$ by year 3.5 , with disturbance growth occurring primarily during years 1.5-3.5, the growth phase of El Niño conditions. During most of the rest of the cycle, a persistent decay of the disturbance occurs, so that the full-period disturbance amplification at any point on the UPO is $\left|\Lambda_{1}\right| \approx 1.8$; the final points of each curve are the full-period am-

FIG. 5. (a) Local 3-month amplification of the unstable (solid line) and neutral (dashed) eigenvectors along the UPO for the standard norm. (b) Cumulative disturbance growth along UPO for initial disturbances $\mathbf{u}_{1}$. (c) Cumulative disturbance growth along UPO for initial disturbances $\mathbf{u}_{2}$.
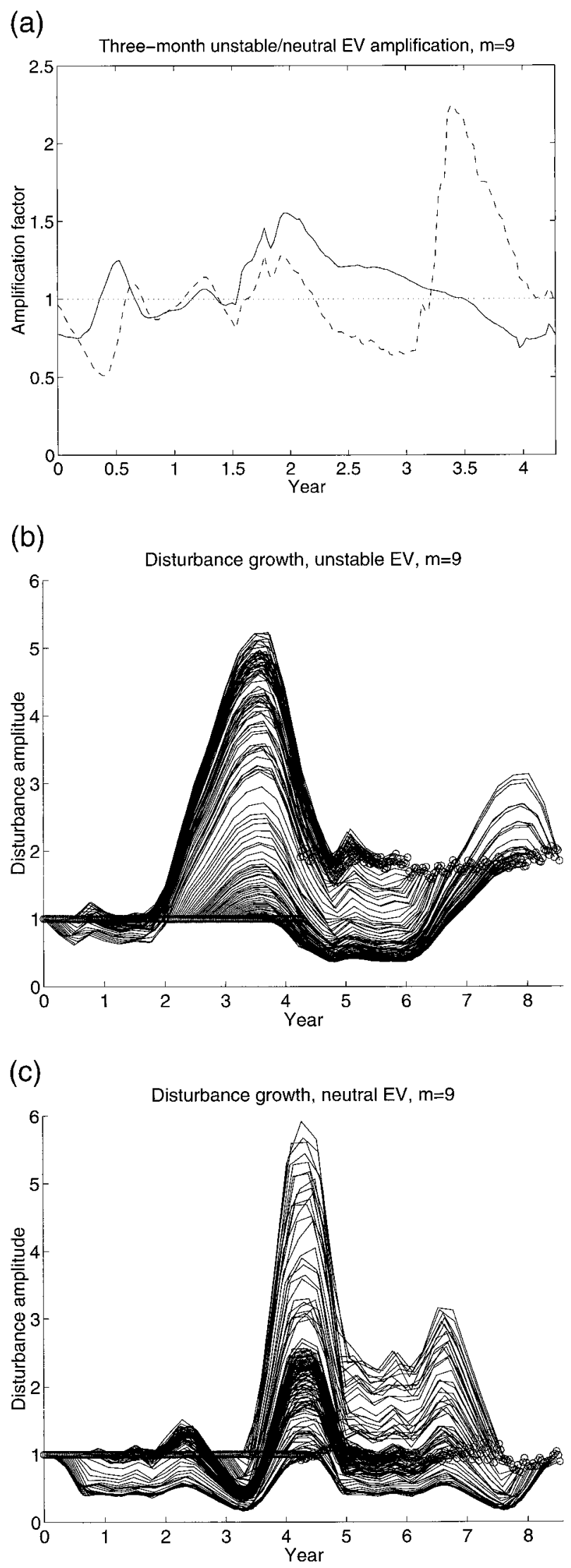
plifications discussed above. For random initial disturbances along the unstable eigenvector at random points along the UPO, the local amplification will in the mean tend to result in preferential appearance of finite amplitude disturbances and disruption of the basic periodic cycle during the growth phase of El Niño conditions. We refer to the corresponding local loss of predictability as the growth-phase predictability barrier.

The cumulative linear growth of disturbances along the neutral eigenvector is dominated by rapid amplification by a factor of 3-5 during the period immediately following the El Niño maximum (Fig. 5c). During most of the rest of the cycle, the neutral disturbances decay. The full-period neutral disturbance amplification factor is $\Lambda_{2} \approx 1$, as above. The amplification of disturbances along the neutral vector following the El Niño maximum is a second mechanism for local predictability loss. If the initial disturbance is sufficiently large, nonlinear effects may lead to a disruption of the basic cycle in this case also. To linear order, the growth of perturbations along the neutral vector represents a phase shift along the cycle, and the corresponding linear loss of predictability is in the timing of events, rather than in the essential character of the oscillation. From a practical point of view, predictability of the timing of ENSO events is just as important as that of the amplitude of the cycle, so the loss of predictability along the direction of the neutral vector is important. We find the neutral vector predictability to be especially small during the El Niño termination phase, indicating that this mechanism may limit our ability to predict the timing of the termination of ENSO events.

The first EOF amplitude dominates both the unstable and neutral eigenvectors of the maps representing the linearized dynamics during the 4.3-yr UPO (Fig. 4). This first EOF has the spatial structure of a mature El Niño event, indicating that the growth mechanism of the disturbance is likely to be similar to that of the mechanism due to which the mature El Niño develops. Another indication that the disturbances grow due to a similar physical mechanism as the El Niño event itself is as follows. The neutral eigenvector is proportional to the rate of change along the UPO itself, and so directly represents the dynamics of the 4.3-yr oscillation. During much of the period of disturbance growth (e.g., 1.5-3 yr in Fig. 1), the unstable eigenvector is roughly parallel to the neutral eigenvector (Fig. 4c).

This similarity of the unstable and neutral eigenvectors during the growth phase, which itself is coincident with the growth phase of El Niño conditions during the 4.3-yr cycle, suggests that the dominant physical mechanism driving the growth of perturbations to the ENSO cycle is nearly the same mechanism that is responsible for the growth of El Niño conditions themselves. As a simple example of how this can occur, consider a linear system undergoing exponential growth: the difference between two solutions will grow exponentially at exactly the same rate, and by the same mechanism, as each solution itself. The growth of disturbances in the present case is clearly more complex than the simple exponential example. During the later part of the El Niño growth phase (2.5-3 yr), the unstable vector continues to amplify while the neutral vector decays (Fig. 5a). However, examination of the eigenvectors (Figs. 4a,b) suggests that the sustained growth of the unstable vector during this period occurs because the unstable vector retains the phase relationship between EOFs 1-3 that supports growth of both vectors near year two. It is this extended period of growth, not a second distinct instability mechanism, that allows the unstable vector to overcome the decay that it suffers over most of the rest of the cycle, and achieve net growth over one full period.

\section{b. Singular vectors}

In this section, we extend the local predictability analysis to include the possibility of rapid transient growth associated with nonnormality of the disturbance dynamics. In the previous section, we analyzed local predictability along the UPO by separately computing the local amplification of disturbances along the unstable and neutral eigenvectors of the full-period return maps. These eigenvectors are not orthogonal in the standard norm (Fig. 4c). Thus, as is generally the case for nonnormal systems, the transient growth of disturbances consisting of superpositions of these two eigenvectors can exceed the rate of growth of either eigenvector alone. Since both eigenvectors are locally tangent, in a general sense, to the model's attractor (Fig. 3), their superpositions lie on, or very close to, the attractor. These superpositions can therefore be interpreted as errors in the specification of the initial position of the state on the attractor, and so are appropriate for consideration in the context of the deterministic prediction problem we study here.

We compute the disturbance that grows most rapidly for a given time interval by singular vector (optimal growth) analysis in which, for each optimization interval, the combination of the unstable and neutral vectors that amplifies most rapidly is calculated by solving an eigenvalue problem. We restrict the optimal growth analysis to the subspace spanned by the unstable and neutral vectors because these are the only eigenmodes for which we have reliable estimates. In general, stable modes often contribute importantly to singular vector structure, and we anticipate that the same would be true here if a more general singular vector calculation were carried out. Thus, the results of our singular vector calculation must be interpreted cautiously and with these restrictions in mind. On the other hand, it could be argued that the present restriction on singular vector structure is appropriate for the study of predictability when the variability is dominated by the dynamical evolution of the model. In that case, the system state will always remain on the attractor, and since stable eigenvectors typically point away from an attractor, model states that 
are reached by superpositions of general stable and unstable modes are likely to lie off the attractor and should be excluded from consideration. Such states can be reached through external forcing or reinitialization, but not through the deterministic evolution of the model.

We calculate singular vectors for the 3-month $\left(n_{m}=\right.$ 9) partial-period maps $\mathbf{M}^{p}$, in the subspace of the unstable $\left(\mathbf{u}_{1}\right)$ and neutral $\left(\mathbf{u}_{2}\right)$ eigenvectors of the composite full-period return map $\mathbf{M}^{c}$, as follows. Let $\mathbf{x}\left(t_{k}\right)$ be a general unit-norm disturbance at time $t_{k}$. Then the linear image at time $t_{k}+m$ of this disturbance is $\mathbf{y}\left(t_{k}\right.$ $+m)=\mathbf{M}^{p}\left(t_{k} ; t_{k}+m\right) \mathbf{x}\left(t_{k}\right)$. To find the disturbance whose norm grows fastest over the 3 months, we maximize the square of the final-time norm,

$$
\|\mathbf{y}\|^{2}=\mathbf{y}^{\mathrm{T}} \cdot \mathbf{y}=\mathbf{x}^{\mathrm{T}}\left(\mathbf{M}^{p}\right)^{\mathrm{T}} \mathbf{M}^{p} \mathbf{x},
$$

with respect to $\mathbf{x}$, subject to the constraint $\|\mathbf{x}\|=1$. This yields the standard singular-vector eigenvalue problem,

$$
\mathbf{L x}=\alpha^{2} \mathbf{x}, \quad \text { and } \quad \mathbf{L}=\left(\mathbf{M}^{p}\right)^{\mathrm{T}} \mathbf{M}^{p},
$$

where the eigenvalues $\alpha$ are the singular values, and the eigenvectors $\mathbf{x}$ are the singular vectors. To restrict to the subspace spanned by $\mathbf{u}_{1}$ and $\mathbf{u}_{2}$, we require that

$$
\mathbf{x}\left(t_{k}\right)=c_{1} \mathbf{u}_{1}\left(t_{k}\right)+c_{2} \mathbf{u}_{2}\left(t_{k}\right)
$$

where $c_{1}$ and $c_{2}$ are constants, chosen such that $\|\mathbf{x}\|=$ 1 but otherwise arbitrary. Then (8) can be rewritten as an eigenvalue problem with the same singular values,

$$
\mathbf{L}_{u} \mathbf{c}=\alpha^{2} \mathbf{c}, \quad \text { and } \quad \mathbf{L}_{u}=\left(\mathbf{U}^{\mathrm{T}} \mathbf{U}\right)^{-1} \mathbf{U}^{\mathrm{T}} \mathbf{L U},
$$

where $\mathbf{c}=\left[c_{1}, c_{2}\right]$ is the eigenvector, and $\mathbf{U}=\left(\mathbf{u}_{1}, \mathbf{u}_{2}\right)$ is the matrix whose columns are the unstable and neutral vectors. We solve the $2 \times 2$ matrix eigenvalue problem (10) using standard numerical methods, for each point $t_{k}$ along the UPO. Since we use the same norms for the initial and final disturbances, the singular values $\alpha$ may be interpreted as amplification factors, and compared directly to the eigenvector amplification factors computed in the preceding section.

The pattern of leading 3-month amplification factors (singular values) for the standard norm (Fig. 6a) is broadly similar to the 3-month eigenvector-amplification patterns examined in the previous section (Fig. 5a, also shown again in Fig. 6a). There is, again, strong disturbance growth during and immediately after the El Niño growth phase, from 1.5-4 yr. Significant differences in the patterns are also apparent. The leading singular value is several times larger than the unstable

FIG. 6. (a) Leading 3-month singular value (thick solid line) in the unstable-neutral subspace (span of $\mathbf{u}_{1}$ and $\mathbf{u}_{2}$ ) along the UPO for the standard norm. The local amplifications from Fig. 5a of the unstable (thin solid) and neutral (dashed) eigenvectors are shown for comparison. (b) The $\mathbf{u}_{1}$ (solid line) and $\mathbf{u}_{2}$ components of the leading 3month singular vector along the UPO for the standard norm. (c) Cumulative disturbance growth along the UPO for initial disturbances equal to the leading 3-month singular vector. (a)

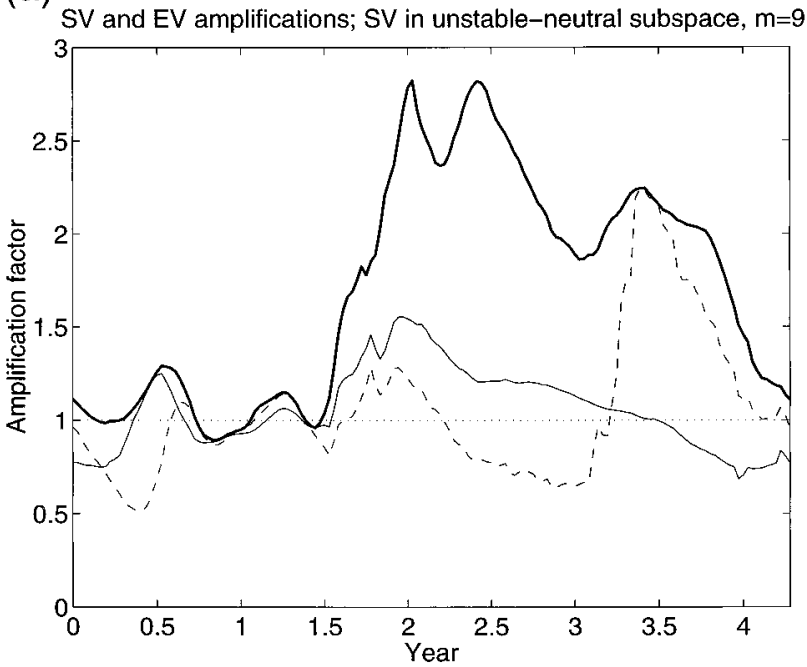

(b)

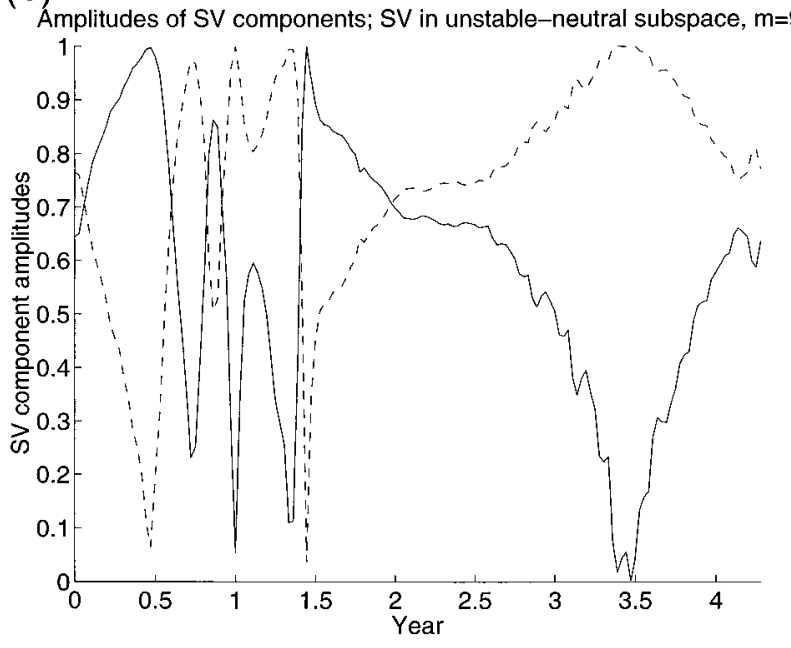

(c)

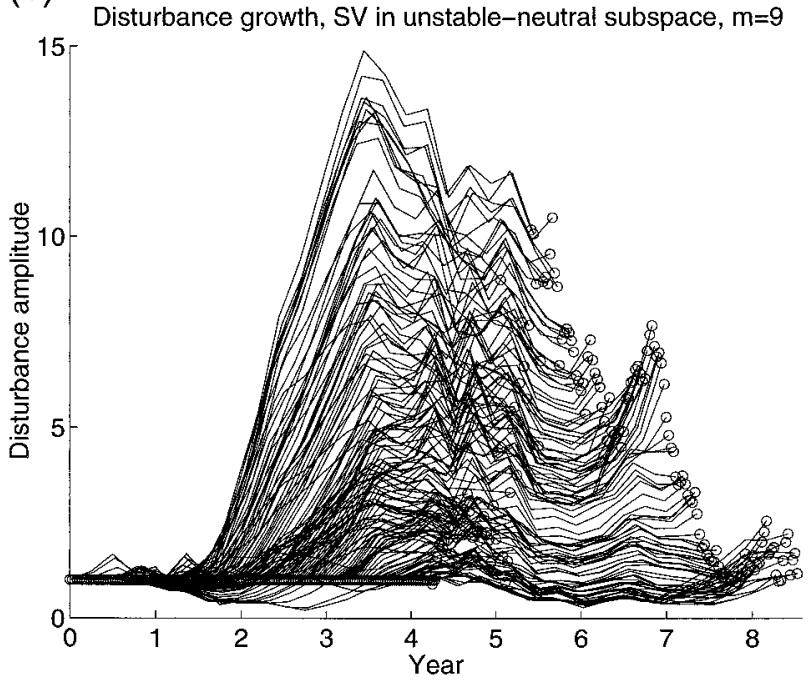


eigenvector amplification over the entire El Niño growth phase. This enhanced disturbance amplification arises from the nonorthogonality of the unstable and neutral eigenvectors during this period, (Figs. 6b, 4c; Moore and Kleeman 1996, 1997). At the El Niño maximum ( $3.5 \mathrm{yr})$, the leading singular vector is dominated by the neutral eigenvector (Fig. 6b), and the leading singular value is essentially equal to the neutral eigenvector amplification. The leading singular value is always at least as large as the largest eigenvector amplification, as it must be, and an amplifying singular vector (singular value greater than one) is found during almost the entire cycle, with exceptions only near $0.5,1$, and $1.5 \mathrm{yr}$. The cumulative amplifications of initial unit-amplitude disturbances corresponding to the 3-month singular vectors at each time along the UPO are shown in Fig. 6c. These are computed as above (Fig. 5c) by computing successive images of the initial disturbance using the partialperiod maps $\mathbf{M}^{p}$. They again emphasize the tendency for sustained disturbance growth during the El Niño growth phase.

Our calculated amplification factors are substantially smaller than those computed by Moore and Kleeman (1996) for the La Niña-El Niño transition period in their intermediate-complexity coupled model. Note, however that besides the fact that our analysis applies to a chaotic model regime and theirs to a regular oscillation, we limit the subspace of optimal perturbations to the neutral and unstable vectors only. Much larger amplifications might be obtained if stable vectors were included.

Chen et al. (1997) found the temporal structure of the singular values in their model to depend quite sensitively on the basic state about which predictability was studied (be it seasonal cycle, or ENSO with or without a seasonal cycle included in the model dynamics). The spatial structure of the singular vectors they found was, in contrast, very robust, and quite dramatically different from both the very small scale structure found by Moore and Kleeman (1996) and in particular from the structure of the ENSO mode itself. Their calculation of optimal structures which, unlike ours, was not restricted to lie on the model attractor, resulted in large-scale zonal structures that are not realizable during the normal model ENSO evolution. This strengthens the motivation for our procedure of limiting the analysis to the subspace of the stable and neutral vectors, which spans the states accessible by the deterministic model dynamics in the absence of external noise. On the other hand, despite the differences in model formulation, basic-state, and methodology, the dependence of leading singular value on ENSO phase found by Chen et al. (1997) is roughly consistent with the present results: their leading singular values are largest during the transition to the growth and decay phases of El Niño but some tendency for disturbance growth persists throughout the growth phase. Our results suggest that, despite the large number of degrees of freedom that in principle contribute to the singular vector calculation, the corresponding amplifi- cations may be traced to the influence of only two modes: the unstable and neutral modes, or in the stable case examined by Chen et al. (1997), the neutral and least-stable damped modes. This hypothesis is supported by the structure of the "final-time" disturbances into which the singular vectors computed by Chen et al. (1997) evolve, which closely resemble the present EOF 1 , the dominant component of both the unstable and neutral modes in our calculations. In addition, we can hypothesize on the basis of our results that in the study of Chen et al. (1997), the disturbance growth during the transition to the growth phase is primarily related to the least-stable damped mode, while that found during the transition to the decay phase is primarily related to the neutral mode.

As discussed above for the local amplification analysis of the eigenvectors, the singular vectors depend on the choice of norm, and substantially different results could be obtained for substantially different norms. For other physically reasonable norms we have examined, the results are somewhat different from the results for the standard norm, but not drastically so, indicating that the growth-phase barrier may be a reasonably robust result.

\section{Conclusions}

When the CZ-intermediate-coupled ENSO anomaly model is run with a specified perpetual July background climatology, it exhibits sustained, weakly chaotic ENSO-cycle oscillations (Tziperman et al. 1995, 1997). During extended periods, the model state tends to follow approximately an unstable, nonlinear, exactly periodic 4.3-yr cycle (UPO). In the present study, we have analyzed the time-dependent modes of linear stability of this cycle, and inferred local predictability properties from this analysis. The analysis is based on linearizing the dynamics around the 4.3-yr cycle in a reconstructed phase space based on the multivariate EOF amplitudes. We can then interpret these results with regard to predictability of ENSO as a function of the ENSO phase. It should be noted that our analysis focuses entirely on this single, lowest-order cycle. Some of the conclusions could be modified by analysis of higher-order cycles. However, examination of the original EOF time series and previous experience with other weakly chaotic models suggests that substantial insight into the model dynamics can be obtained from the analysis of this single cycle.

We found unstable and neutral eigenmodes of the 4.3yr cycle that have time-dependent spatial structure. We also found that, consistent with recent studies that focus on the nonnormal aspects of ENSO dynamics, (Farrell and Ioannou 1996; Penland and Sardeshmukh 1995; Moore and Kleeman 1996, 1997; Chen et al. 1997) the time-dependent eigenmodes were not orthogonal (in a standard norm, based on the EOF amplitudes). Consequently, we analyzed the local growth of linear distur- 
bances using singular vectors (optimal disturbances; Farrell and Ioannou 1996) as well as eigenmodes.

Both the eigenmode and the restricted singular vector analyses we have performed suggest that there is a predictability barrier associated with the growth phase of El Niño conditions. That is, ENSO's predictability is smallest during the growth phase of El Niño conditions in the equatorial east Pacific. This is our main result, and we termed this variation of predictability along the ENSO cycle the growth-phase predictability barrier. The implication is that ENSO will be least predictable exactly at the time when its predictability is of most interest from a practical point of view, during the development of El Niño conditions. These results are roughly consistent with those of Moore and Kleeman (1996) who have analyzed a nonchaotic, periodic, self-sustained ENSO solution in another intermediate coupled ENSO model.

The growth-phase predictability barrier evidently arises because the growth mechanism for disturbances to the cycle is nearly the same as the growth mechanism for the El Niño conditions themselves. In our phasespace representation, both the basic state (the nonlinear 4.3-yr periodic solution whose predictability we examine) and the disturbances to the basic state are represented by the EOF amplitudes. The first EOF amplitude dominates both the unstable and neutral eigenvectors of the maps representing the linearized dynamics during the 4.3-yr UPO (Fig. 4). This first EOF has the spatial structure of a mature El Niño event, indicating that the growth mechanism of the disturbance is likely to be similar to that of the mechanism due to which the mature El Niño develops. Other indications that the disturbances grow due to a similar physical mechanism as the El Niño event itself have been identified as well.

The absence of a second instability mechanism for the disturbances essentially requires disturbance growth to occur during the growth phase of the basic cycle, leading to the growth-phase predictability barrier. This reasoning does not explain why a second predictability barrier does not arise in connection with the growth of La Niña conditions, during the opposite phase of the cycle. The physical origin of this asymmetry most likely lies in the asymmetry in the physical processes responsible to the growth of El Niño and La Niña. While both are manifestations of the coupled ocean atmospheric instability, there are some instability-enhancing processes that are active only during El Niño, such as heating in the central Pacific that depends on the absolute sea surface temperature being above some threshold (Moore and Kleeman 1996).

The finding that the most rapidly growing perturbations have the same structure and physical mechanism as the mature El Niño conditions seemingly contradicts a recent estimate of the optimal disturbances in another intermediate ENSO model (Moore and Kleeman 1997) where the optimal structures seemed concentrated in the west Pacific. On the other hand, several additional stud- ies have recently evaluated the optimal structures of the equatorial Pacific, and many of these have come up with optimal structures that are centered in the east Pacific, consistent with our finding (that is based on quite a different approach) here. The restriction of the singular vectors in our case to the unstable-neutral subspace could also have significantly affected their spatial structure.

A number of previous studies have analyzed the seasonal dependence of ENSO predictability, often finding the spring predictability barrier (Webster and Yang 1992; Torrence and Webster 1998; Weiss and Weiss 1999; Xue et al. 1994; Moore and Kleeman 1996). The spring predictability barrier has been explained in terms of the seasonal dependence of the air-sea coupling process, due to the seasonal movement of the Intertropical Convergence Zone and other factors. The spring is also, of course, the period when the El Niño growth phase tends to occur (Tziperman et al. 1998; Galanti and Tziperman 2000). A statistical analysis of the spring predictability barrier from predictability studies based on actual observed ENSO events is likely to include springs from both El Niño years and non-El Niño years. It is well understood that the seasonal background of the equatorial Pacific results in a springtime predictability barrier. However, one wonders to what degree the spring predictability barrier found in analyses based on observed ENSO events may actually be a manifestation of the growth-phase predictability barrier. To find out, one might perform a study of the seasonal predictability separately for El Niño years and non-El Niño years.

The CZ model using a perpetual July background is clearly a highly simplified representation of the ENSO dynamics. The restriction to a nonseasonal model in the present study was essential for the objectives of this study, in order to be able to differentiate predictability dependence on the phase of the ENSO cycle from the predictability dependence on the phase of the seasonal cycle. Additionally, a chaotic attractor is composed of an infinite set of UPOs, and we have restricted our analysis to a single, though dominant, UPO of the CZ model. We feel that the main result reported here-the existence of an ENSO predictability barrier that is tied to the growth phase of El Niño conditions-is likely to be robust. This belief is based on our identification of the physical mechanisms that limit the predictability during the growth phase. That the physical mechanism limiting predictability along a nonlinear periodic solution is essentially the same mechanism driving the oscillation itself, is a result that depends primarily on the weakness of the large-scale instabilities of the ENSO cycle, relative to the strength of the interactions associated with the evolution of the cycle itself. [A similar situation has recently been described in an analysis of the predictability of baroclinic waves in a weakly nonlinear channel model (Samelson 2001)].

These general characteristics of the model ENSO cycle are likely to be more robust than the particular model 
we investigate. In any case, analyzing predictability along additional UPOs and extending our approach to the seasonally forced case are important future steps that will help to put our results in a more general context. One of our objectives was to find a predictability signal that might differentiate the chaotic regime of ENSO from the nonchaotic one. However, the rough correspondence between our results with those of Moore and Kleeman (1996) who analyzed a regular, nonchaotic oscillation indicates that we need to keep looking for such a differentiating signal in order to understand whether the observed irregularity of El Niño arises primarily from stochastic forcing or from low-order chaos.

Acknowledgments. This work was partially supported for Roger Samelson by the Office of Naval Research, Grant N00014-98-1-0813, and the National Science Foundation, Grant OCE98-96184, and for Eli Tziperman by the Israeli Academy of Sciences. We are grateful to three anonymous reviewers for comments that improved several aspects of the presentation.

\section{REFERENCES}

Artuso, R., E. Aurell, and P. Cvitanović, 1990a: Recycling of strange sets. Part I: Cycle expansions. Nonlinearity, 3, 325-359.

,-- , and $-1990 \mathrm{~b}$ : Recycling of strange sets. Part II: Applications. Nonlinearity, 3, 361-386.

Chang, P., B. Wang, T. Li, and L. Ji, 1994: Interactions between the seasonal cycle and the Southern Oscillation-Frequency entrainment and chaos in a coupled ocean-atmosphere model. Geophys. Res. Lett., 21, 2817-2820.

Chen, D., S. E. Zebiak, A. J. Busalacchi, and M. A. Cane, 1995: An improved procedure for El Niño forecasting: Implications for predictability. Science, 269, 1699-1702.

Chen, Y. Q., D. S. Battisti, T. N. Palmer, J. Barsugli, and E. S. Sarachik, 1997: Study of the predictability of tropical Pacific SST in a coupled atmosphere-ocean model using singular vector analysis: The role of the annual cycle and the ENSO cycle. Mon. Wea. Rev., 125, 831-845.

Christiansen, F., P. Cvitanović, and V. Putkaradze, 1997: Spatio-temporal chaos in terms of unstable recurrent patterns. Nonlinearity, 10, 55-70.

Coddington, E., and N. Levinson, 1955: Theory of Ordinary Differential Equations. McGraw-Hill, 429 pp.

Cvitanović, P., R. Artuso, R. Mainieri, and G. Vatay, 2000: Classical and Quantum Chaos. Niels Bohr Institute, 682 pp. [Available on-line from http://www.nbi.dk/chaosBook/.]

Farrell, B. F., and P. J. Ioannou, 1996: Generalized stability theory. Part I: Autonomous operators. J. Atmos. Sci., 53, 2025-2040.

Galanti, E., and E. Tziperman, 2000: ENSO's phase locking to the seasonal cycle in the fast-SST, fast-wave, and mixed-mode regimes. J. Atmos. Sci., 57, 2936-2950.

Jin, F.-F., D. Neelin, and M. Ghil, 1994: ENSO on the devil's staircase. Science, 264, 70-72.

$\ldots, \ldots$, and —_, 1996: El Niño Southern Oscillation and the annual cycle: Subharmonic frequency-locking and aperiodicity. Physica D., 98, 442-465.

Moore, A. M., and R. Kleeman, 1996: The dynamics of error growth and predictability in a coupled model of ENSO. Quart. J. Roy. Meteor. Soc., 122, 1405-1446.

_ , and — 1997: The singular vectors of a coupled oceanatmosphere model of ENSO. Part II: Sensitivity studies and dynamical interpretation. Quart. J. Roy. Meteor. Soc., 123, 9831006.

Neelin, J. D., D. S. Battisti, A. C. Hirst, F.-F. Jin, Y. Wakata, T. Yamagata, and S. Zebiak, 1998: ENSO theory. J. Geophys. Res. Oceans, 103, 14 261-14 290.

Penland, C., and P. D. Sardeshmukh, 1995: The optimal growth of tropical sea surface temperature anomalies. J. Climate, 8, 19992024.

Samelson, R. M., 2001: Periodic orbits and disturbance growth for baroclinic waves. J. Atmos. Sci., 58, 436-450.

Strong, C., F. F. Jin, and M. Ghil, 1995: Intraseasonal oscillations in a barotropic model with annual cycle, and their predictability. J. Atmos. Sci., 52, 2627-2642.

Torrence, C., and P. J. Webster, 1998: The annual cycle of persistence in the El Niño Southern Oscillation. Quart. J. Roy. Meteor. Soc., 124, 1985-2004.

Tziperman, E., L. Stone, M. A. Cane, and H. Jarosh, 1994: El niño chaos: Overlapping of resonances between the seasonal cycle and the Pacific ocean-atmosphere oscillator. Science, 264, 7274.

- M. A. Cane, and S. E. Zebiak, 1995: Irregularity and locking to the seasonal cycle in an ENSO prediction model as explained by the quasi-periodicity route to chaos. J. Atmos. Sci., 52, 293306.

- H. Scher, S. E. Zebiak, and M. A. Cane, 1997: Controlling spatiotemporal chaos in a realistic El Niño prediction model. Phys. Rev. Lett., 79, 1034-1037.

- M. A. Cane, S. E. Zebiak, Y. Xue, and B. Blumenthal, 1998: Locking of El Niño's peak time to the end of the calendar year in the delayed oscillator picture of ENSO. J. Climate, 11, 21912199.

Vallis, G. K., 1986: El Niño: A chaotic dynamical system? Science, 232, 243-245.

Webster, P. J., and S. Yang, 1992: Monsoon and ENSO: Selectively interactive systems. Quart. J. Roy. Meteor. Soc., 118, 877-926.

Weiss, J. P., and J. B. Weiss, 1999: Quantifying persistence in ENSO. J. Atmos. Sci., 56, 2737-2760.

Xue, Y., M. A. Cane, S. E. Zebiak, and M. B. Blumenthal, 1994: On the prediction of ENSO: A study with a low order Markov model. Tellus, 46A, 512-528.

Zebiak, S. E., and M. A. Cane, 1987: A model El Niño-Southern Oscillation. Mon. Wea. Rev., 115, 2262-2278.

Zoldi, S., and H. Greenside, 1998: Spatially localized unstable periodic orbits of a high-dimensional chaotic system. Phys. Rev. E, 57, R2511-R2514. 